\title{
Optical imaging beyond the diffraction limit via dark states
}

\author{
Hebin Li, ${ }^{1}$ Vladimir A. Sautenkov, ${ }^{1,2}$ Michael M. Kash, ${ }^{1,3}$ Alexei V. Sokolov, ${ }^{1}$ George R. Welch, ${ }^{1}$ Yuri V. Rostovtsev, ${ }^{1}$ \\ M. Suhail Zubairy, ${ }^{1,4}$ and Marlan O. Scully ${ }^{1,5}$ \\ ${ }^{1}$ Department of Physics and Institute for Quantum Studies, Texas A\&M University, College Station, Texas 77843-4242, USA \\ ${ }^{2}$ P.N. Levedev Physical Institute, 53 Leninsky prospect, Moscow 119991, Russia \\ ${ }^{3}$ Department of Physics, Lake Forest College, Lake Forest, Illinois 60045, USA \\ ${ }^{4}$ Texas A\&M University at Qatar, Education City, P.O. Box 23874, Doha, Qatar \\ ${ }^{5}$ Applied Physics and Materials Science Group, Engineering Quad, Princeton University, Princeton, New Jersey 08544, USA
}

(Received 15 March 2008; published 1 July 2008)

\begin{abstract}
We study the possibility of creating spatial patterns having subwavelength size by using the so-called dark states formed by the interaction between atoms and optical fields. These optical fields have a specified spatial distribution. Our experiments in Rb vapor display spatial patterns that are smaller than the length determined by the diffraction limit of the optical system used in the experiment. This approach may have applications to interference lithography and might be used in coherent Raman spectroscopy to create patterns with subwavelength spatial resolution.
\end{abstract}

DOI: 10.1103/PhysRevA.78.013803

PACS number(s): 42.50.Gy, 42.25.Kb, 42.30.Va

\section{INTRODUCTION}

The ability to create small images is important for material processing technology and for improving the resolution of microscopy for biomedical applications [1]. Recently, several methods have been presented that are able to overcome the diffraction limit of the imaging system. Quantum microscopy is based on using a nonclassical optical field approach $[2,3]$. Microscopy with classical fields can be enhanced by the nonlinear optical response of the medium [4]. Classical field amplitude and phase arrangements can be used to locate the position of an atom with subwavelength precision in an atomic beam [5-7] or in a cavity [8]. Localization techniques have been applied to lithography [9-11], and have achieved subwavelength diffraction and imaging using the Dopplerontype resonances $[12,13]$.

Here, we suggest an approach that is based on coherent population trapping [14-18]. Optical fields applied to a three-level quantum system excite the so-called dark state, which is decoupled from the fields. Similar approaches using coherent population trapping have also been developed by several groups (for example, see [19-22]).

As a qualitative introduction, assume that the drive field Rabi frequency $\Omega_{d}$ has the particular spatial distribution sketched in Fig. 1(a) by the solid line (1). The weak probe field Rabi frequency $\Omega_{p}\left(\Omega_{p} \ll \Omega_{d}\right)$ has a diffraction limited distribution [shown by the dashed line (2) in Fig. 1(a)]. The probe and drive fields are applied to the atom (see the inset in Fig. 2, for the case of ${ }^{87} \mathrm{Rb}$ atoms, where $|a\rangle=\mid 5^{2} P_{1 / 2}, F$ $=1, m=0\rangle, \quad|b\rangle=\left|5^{2} S_{1 / 2}, F=2, m=-1\right\rangle, \quad|c\rangle=\mid 5^{2} S_{1 / 2}, F=2, m$ $=+1\rangle$ ). At all positions of nonzero drive field, the dark state, which is given [16] by $|D\rangle=\left(\Omega_{p}|c\rangle-\Omega_{d}|b\rangle\right) / \sqrt{\Omega_{p}^{2}+\Omega_{d}^{2}}$, is practically $|b\rangle$. When the drive field is zero, the dark state is $|c\rangle$, and the atoms at these positions are coupled to the fields and some atoms are in the upper state $|a\rangle$. The size of a spot where the atoms are excited depends on the relaxation rate $\gamma_{c b}$ between levels $|b\rangle$ and $|c\rangle$. For $\gamma_{c b}=0$, the size of spot is zero, smaller than the optical wavelength.

The Hamiltonian of a three-level atom interacting with optical fields (see the inset in Fig. 2) is given by

$$
H=\hbar \Omega_{d}|a\rangle\left\langle b\left|+\hbar \Omega_{p}\right| a\right\rangle\langle c|+\operatorname{adj} .
$$

where $\Omega_{d, p}=\wp_{d, p} E_{d, p} / \hbar$ are the Rabi frequencies of the drive $E_{d}$ and the probe $E_{p}$ fields, respectively; $\wp_{d, p}$ are the dipole moments of the corresponding optical transitions. Then, the atomic response is given by the set of density matrix equations [16]

$$
\dot{\rho}=-\frac{i}{\hbar}[H, \rho]-\frac{\Gamma \rho+\rho \Gamma}{2},
$$

where $\Gamma$ describes the relaxation processes. The propagation of the probe field $\Omega_{p}$ through the cell is governed by Maxwell's equations and, for propagation in the $z$ direction, can be written in terms of the probe field Rabi frequency as

$$
\frac{\partial \Omega_{p}}{\partial z}=-i \eta \rho_{a b}-i \frac{1}{2 k} \frac{\partial^{2}}{\partial x^{2}} \Omega_{p} .
$$

The first term accounts for the dispersion and absorption of the resonant three-level medium, and the second term describes the focusing and/or diffraction of the probe beam. The density matrix element $\rho_{a b}$ is related to the probe field absorption which in turn depends on the detuning and the drive field. This is characterized by an absorption coefficient:

$$
\kappa=\eta \frac{\Gamma_{c b}}{\Gamma_{a b} \Gamma_{c b}+\left|\Omega_{d}(z, x)\right|^{2}},
$$

where $\Gamma_{c b}=\gamma_{c b}+i \omega$ and $\Gamma_{a b}=\gamma+i \omega ; \omega=\omega_{a b}-\nu$ is the detuning from the atomic frequency $\omega_{a b} ; \gamma$ is the relaxation rate at the optical transition; and $\eta=3 \lambda^{2} N \gamma_{r} / 8 \pi ; N$ is the atomic density; $\gamma_{r}$ is the spontaneous emission rate. We now assume that the drive field has a distribution of intensity near its extrema given by 

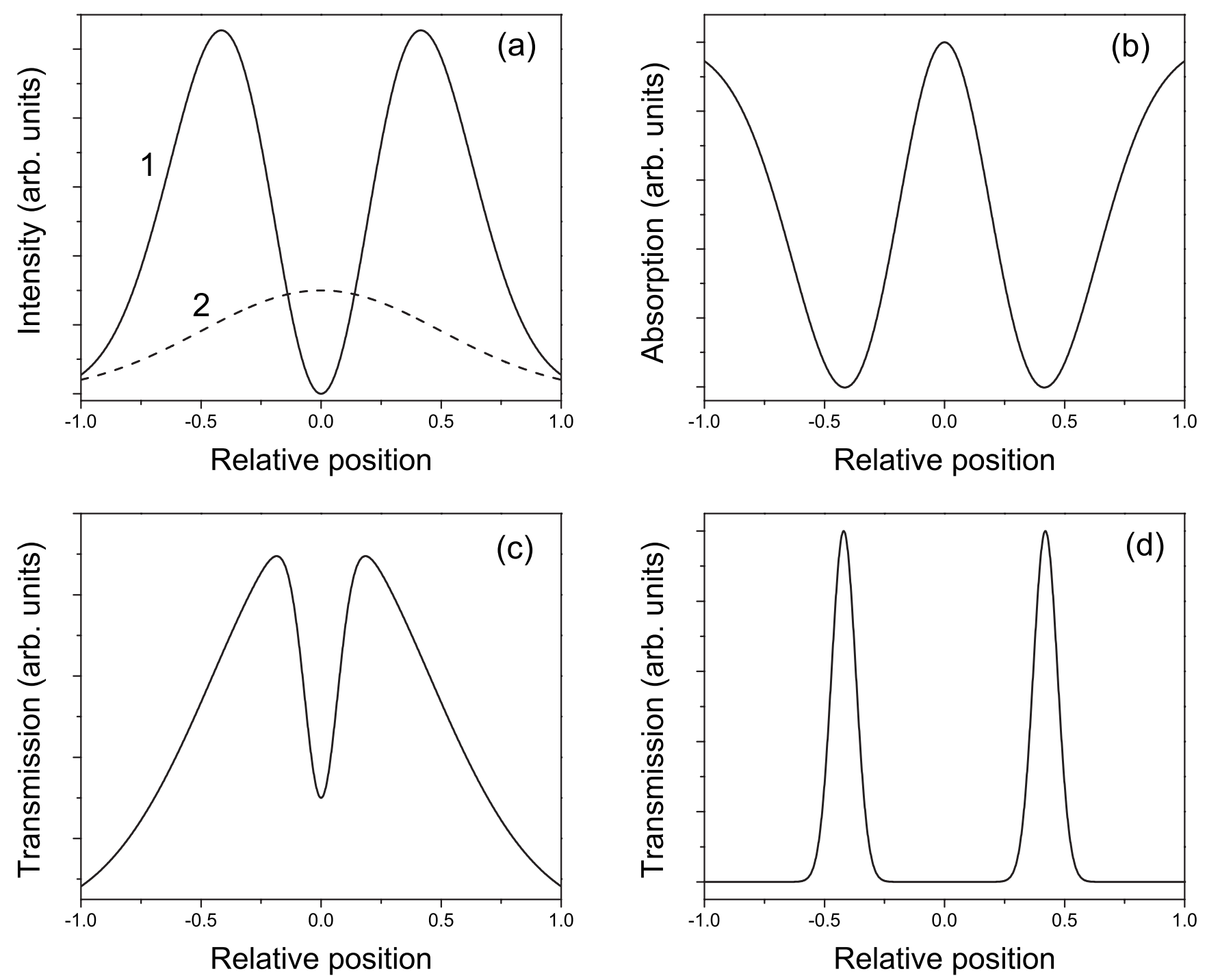

FIG. 1. Qualitative description of the idea. (a) Distribution of the drive (1) and the probe (2) fields vs a transverse spatial coordinate at the entrance to the cell. (b) Dependence of the absorption coefficient given by Eq. (4) vs position. Plots (c) and (d) show the distribution of the probe beam after propagating through the cell. Case (c) is for a strong drive field and relatively low optical density. Case (d) is for a relatively weak drive field and large optical density.

$$
\left|\Omega_{d}(z, x)\right|^{2}=\left|\Omega_{0}\right|^{2} \begin{cases}{\left[1-\left(\frac{x-x_{0}}{L}\right)^{2}\right],} & x \simeq x_{0}, \\ \left(\frac{x}{L}\right)^{2}, & x \ll L,\end{cases}
$$

where $\Omega_{0}=\Omega_{d}\left(z, x_{0}\right), L$ is the separation distance between the peaks of the drive field distribution, and a typical absorption profile vs $x$ is shown in Fig. 1(b). Neglecting the diffraction term in Eq. (3), we can write an approximate solution for Eq. (3) as

$$
\Omega_{p}(z, x)=\Omega_{p}(z=0, x) \exp (-\kappa z) .
$$

For relatively low optical density $\operatorname{Re}(\kappa) z \simeq 1$, nearly all of the probe field propagates through the cell except for a small part where the drive field is zero [see Fig. 1(a)]. Absorption occurs there because the probe beam excites the atomic me- dium. The width of the region of the excited medium, in the vicinity of zero drive field, is characterized by

$$
\Delta x=L \sqrt{\frac{\Gamma_{a b} \Gamma_{c b}}{|\Omega|^{2}}},
$$

where $\Omega=\Omega_{d}(z=0, x=0)$. This region is small, but its contrast is limited because of the finite absorption of the medium at the center of optical line [Fig. 1(c)].

For higher optical density, this narrow feature becomes broadened [compare Fig. 1(c) and 1(d)], but two narrow peaks are formed during the propagation of the probe beam [see Fig. 1(d)]. For zero detuning, their width is given by

$$
\Delta x=L \sqrt{\frac{|\Omega|^{2}}{\eta \gamma_{c b} z}} .
$$

The drive field provides flexibility for creating patterns with sizes smaller than the wavelength of the laser. The dis- 


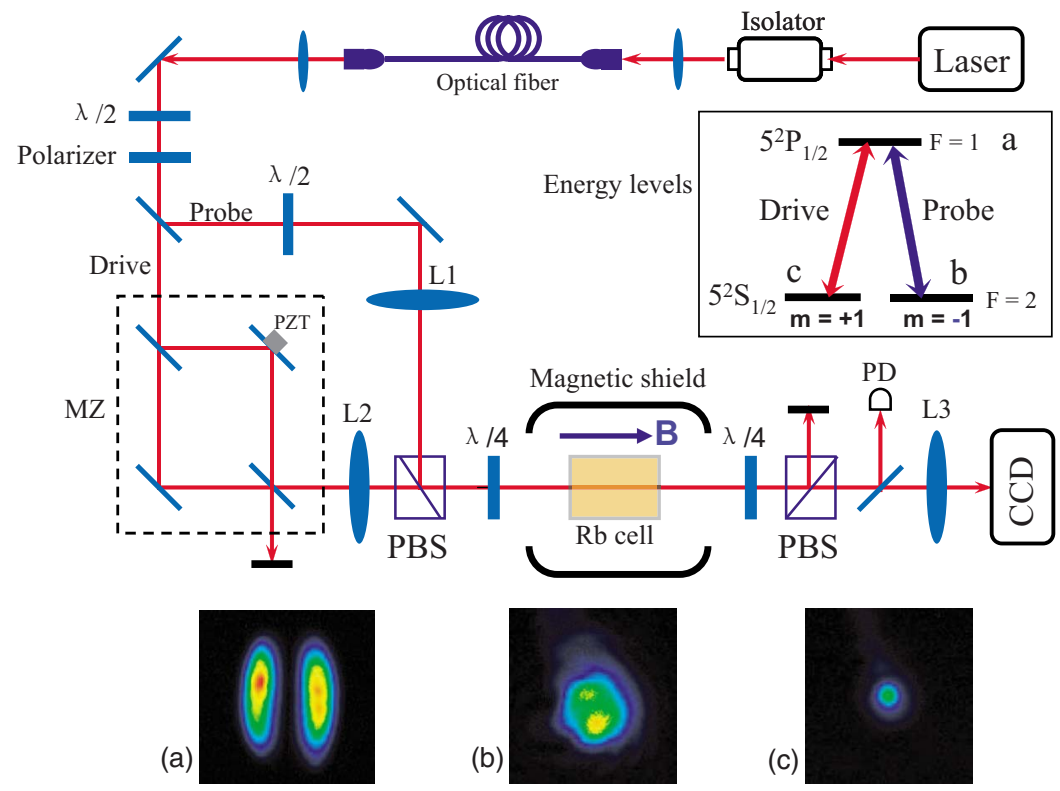

FIG. 2. (Color online) Experimental schematic. $\lambda / 2$ : half-wave plate; $\lambda / 4$ : quarter-wave plate; L1, L2, L3: lenses; MZ: Mach-Zehnder interferometer; PZT: piezoelectric transducer; PBS: polarizing beam splitter, PD: photodiode; CCD: CCD camera. Picture (a) is the spatial intensity distribution of the drive field. Picture (b) is the beam profile of the parallel probe beam without the lens L1. Picture (c) is the beam profile of the diffraction limited probe beam with the lens L1. All three of pictures have been made with the camera at the location of the cell, which has temporarily been removed. The inset is the energy diagram of the $\mathrm{Rb}$ atom, showing representative sublevels.

tribution of fields is governed by electrodynamics and has a diffraction limit, while the distribution of molecules in their excited states is NOT related to the diffraction limit, but rather determined by the relaxation rates $\Gamma_{a b}$ and $\Gamma_{c b}$, and thus can have spatial sizes smaller than the wavelength.

\section{EXPERIMENTAL SETUP AND RESULTS}

In this paper, we report a proof-of-principle experiment in $\mathrm{Rb}$ vapor to demonstrate our approach. We have observed that the distribution of the transmitted probe beam intensity has a double-peak pattern, which is similar to that of the drive beam, but the width of the peaks of the probe beam is narrower than that of the drive beam.

The experimental schematic is shown in Fig. 2. We obtain a good quality spatial profile by sending the radiation of an external cavity diode laser through a polarization-preserving single-mode optical fiber. The laser beam is vertically polarized and split into two beams (drive and probe). The probe beam carries a small portion of the laser intensity, and its polarization is rotated to be horizontal.

To create a double-peak spatial distribution for the drive field, the drive beam is split into two beams that cross at a small angle, using a Mach-Zehnder interferometer (shown in the dashed square of Fig. 2). A typical two-peak interference pattern of crossing beams is shown as Fig. 2(a).

The probe and drive beams combine on a polarizing beam splitter, arranged so that the probe field and the interference pattern of the drive field are overlapped in a $\mathrm{Rb}$ cell. The $\mathrm{Rb}$ cell has a length of $4 \mathrm{~cm}$, and is filled with ${ }^{87} \mathrm{Rb}$. A magnetic shield is used to isolate the cell from any environmental magnetic fields, while a solenoid provides an adjustable, longitude magnetic field. The cell is installed in an oven that heats the cell to reach an atomic density of $10^{12} \mathrm{~cm}^{-3}$. The laser is tuned to the $\mathrm{D}_{1}$ line of ${ }^{87} \mathrm{Rb}$ at the transition $5^{2} \mathrm{~S}_{1 / 2}(F=2) \rightarrow 5^{2} \mathrm{P}_{1 / 2}(F=1)$.

As stated above, the probe and drive beams have the orthogonal linear polarizations. A quarter-wave plate converts them into left and right circularly polarized beams, which couple two Zeeman sublevels of the lower level and one sublevel of the excited level of the $\mathrm{Rb}$ atoms (see the inset of Fig. 2).

After passing through the cell, the probe and drive beams are converted back to linear polarizations by another quarterwave plate and the separated by a polarizing beam splitter. The power of transmitted probe field is monitored by a photodiode. The spatial intensity distribution of probe field is recorded by an imaging system, consisting of the lens L3 and a CCD camera.

The intensity of the probe beam is low enough that its transmission through the cell is almost zero without the presence of drive laser. Applying the drive laser makes the atomic medium transparent for the probe laser wherever the EIT condition is satisfied. If the drive laser has a certain transverse spatial distribution, then that pattern can be projected to the transmission profile of the probe laser.

Two different experiments have been performed. In the first experiment, the lenses L1 and L2 are not used, and the probe beam is a parallel beam with a diameter of $1.4 \mathrm{~mm}$. The image of the drive intensity distribution in the cell is shown in Fig. 3(a). The probe intensity has a Gaussian distribution before entering the cell, and its distribution is similar to the drive intensity distribution after the cell. As shown in Fig. 3(b), however, the transmitted probe intensity has a distribution that has sharper peaks compared with the pattern of the drive intensity. The horizontal cross sections of the drive and the transmitted probe distributions are shown in Figs. 3(c) and 3(d), respectively. In the drive intensity profile, the width (FWHM) of the peaks is $0.4 \mathrm{~mm}$. The width (FWHM) of the peaks in the transmitted probe intensity profile is $0.1 \mathrm{~mm}$. The spacing between two peaks is the same for both the drive and transmitted probe fields. We define the finesse as the ratio of the spacing between peaks to the width of peaks. The finesse of the transmitted probe intensity distribution is a factor of 4 smaller than that of the drive intensity distribution.

In the second experiment, the lenses L1 and L2 are used. A parallel probe beam [Fig. 2(b)] with a diameter of $1.4 \mathrm{~mm}$ 

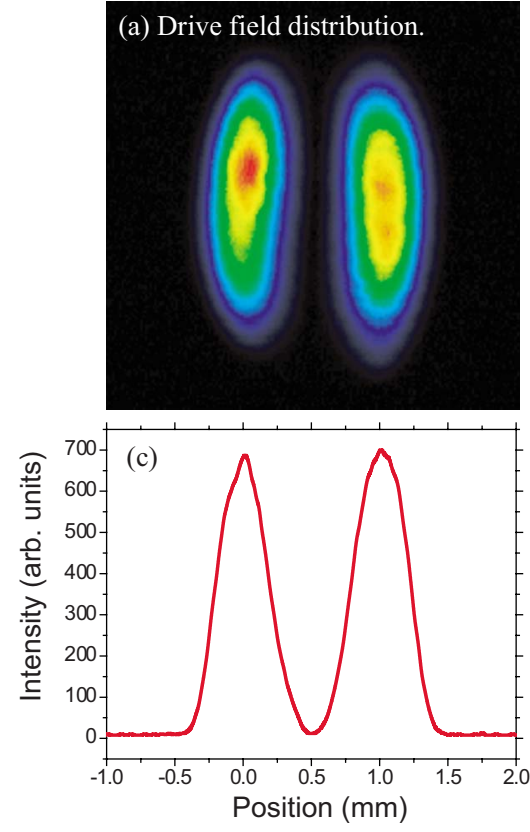
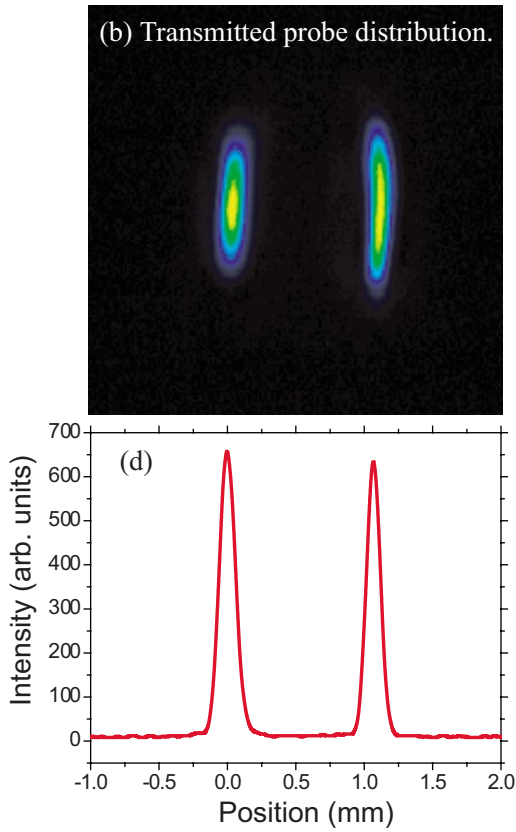

FIG. 3. (Color online) The results of the experiment with a parallel probe beam. Picture (a) shows the image of the intensity distribution of the drive field in the $\mathrm{Rb}$ cell. Picture (b) shows the intensity distribution of the transmitted probe field. Curves (c) and (d) are the corresponding intensity profiles. The widths of the peaks in curves (c) and (d) are $0.4 \mathrm{~mm}$ and $0.1 \mathrm{~mm}$, respectively. is focused by the lens L1, which has a focal length of $750 \mathrm{~mm}$. The beam size at the waist is $0.5 \mathrm{~mm}$, which is diffraction limited. To assure experimentally that the beam is diffraction limited, we increased the beam diameter of the parallel beam by the factor of 2 , and the beam size at the waist became two times smaller. The lens L2 is used to make the drive beam smaller in the Rb cell, where the pattern of drive field is spatially overlapped with the waist of the probe beam. Classically, there should be no structures at the waist of the probe beam because it is diffraction limited. Structures can be created in a region smaller than the diffraction limit in our experiment, however. The experimental result is shown in Fig. 4. The drive field still has a double peak intensity distribution [Fig. 4(a)]. The transmission of the diffraction limited probe beam also has a double-peak intensity distribution as shown in Fig. 4(b). Curves (c) and (d) are the beam profiles of the drive and transmitted probe beams, respec- tively. The width (FWHM) of the peaks in the drive beam is $165 \mu \mathrm{m}$, and the width (FWHM) of the peaks in the transmitted probe beam is $93 \mu \mathrm{m}$. The finesse of the transmitted probe beam is 1.8 times greater than that of the drive beam. For the probe beam, the structure created within the diffraction limit has a size characterized by the width of peaks (93 $\mu \mathrm{m})$. This characteristic size is 5 times smaller than the size of the diffraction limited probe beam $[500 \mu \mathrm{m}$, see the spot of Fig. 2(c)].

\section{DISCUSSION}

Thus, we have demonstrated that our concept works in $\mathrm{Rb}$ vapor. Although the diffraction limit is "beaten," the experiment does not violate any laws of optics. The probe beam is diffraction limited, but the atoms are much smaller than the size of diffraction-limited beam. Moreover, due to the strong
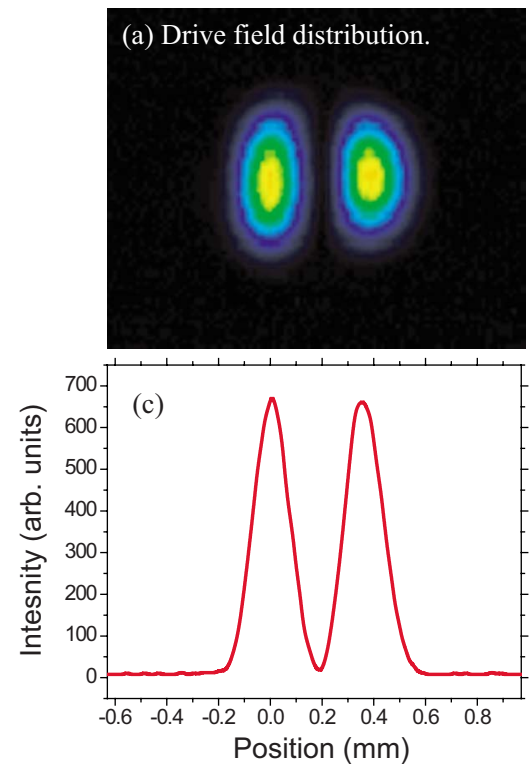
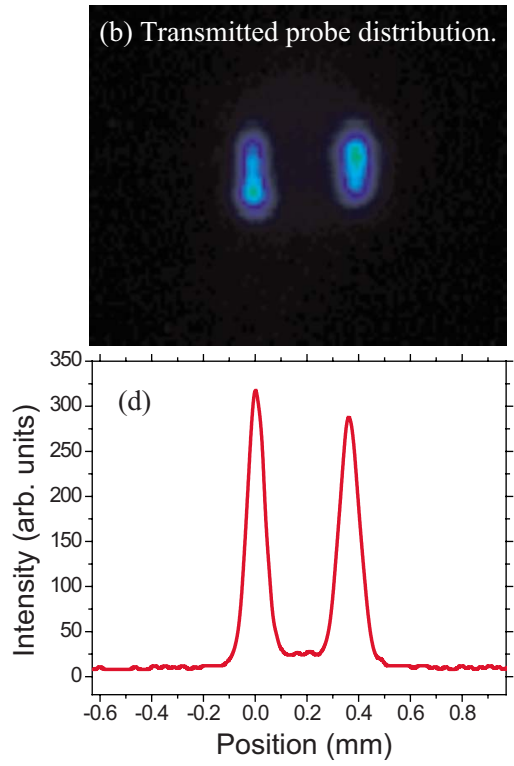

FIG. 4. (Color online) The results of the experiment with the diffraction limited probe beam. Picture (a) shows the image of the intensity distribution of the drive field in the Rb cell. Picture (b) shows the image of the intensity distribution of the transmitted probe field. Curves (c) and (d) are the corresponding profiles. The widths of the peaks in curves (c) and (d) are $165 \mu \mathrm{m}$ and $93 \mu \mathrm{m}$, respectively. 

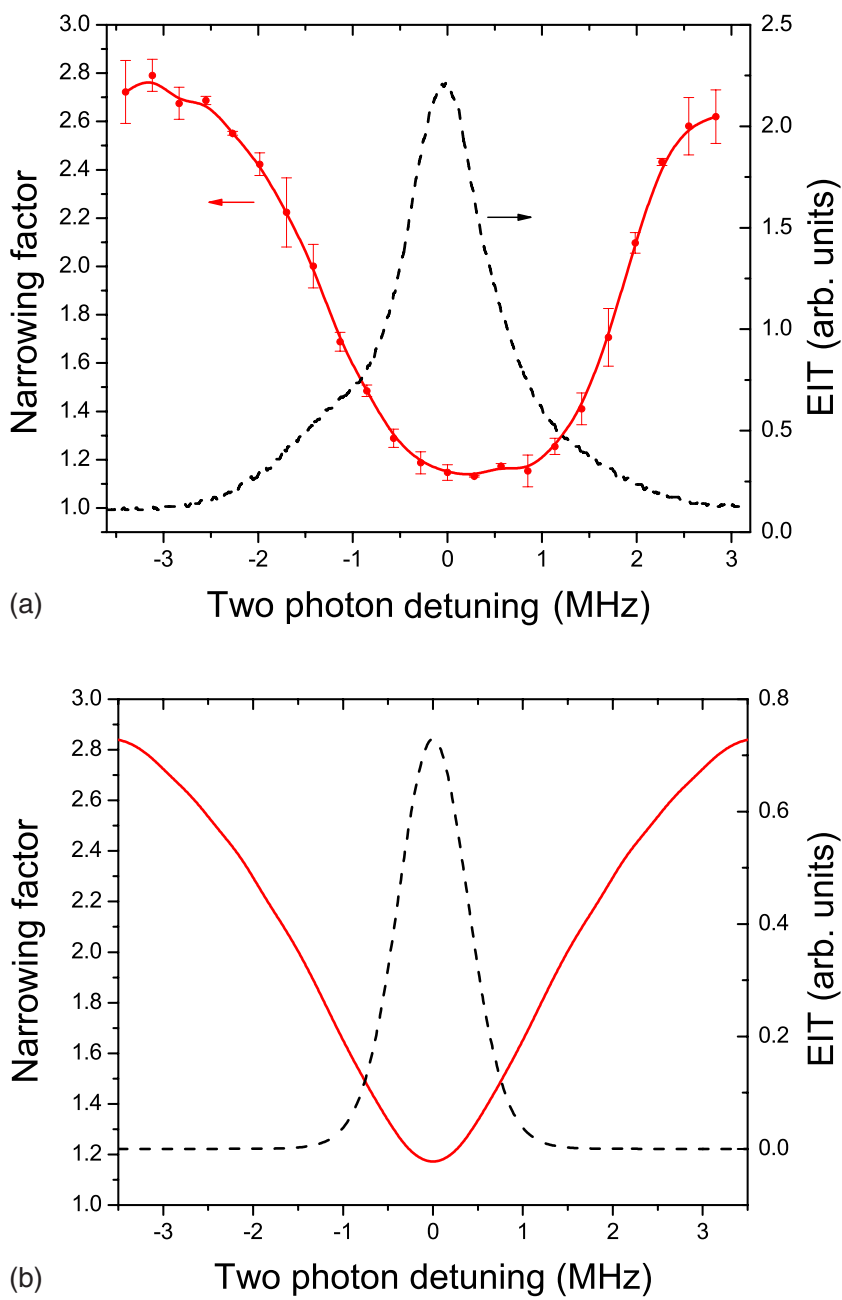

FIG. 5. (Color online) Narrowing of the transmitted probe intensity distribution as function of the probe detuning: (a) experimental results, and (b) theoretical simulation. The transmitted probe profile is shown as well.

nonlinearity of the EIT, the characteristic size of the pattern in the transmitted probe beam is much smaller than that of the drive beam and the diffraction limit of the probe beam.

We have also measured the narrowing effect vs the detuning of the probe field and have performed simulations using the density matrix approach. The results are shown in Fig. 5.
The calculations reproduce the data satisfactorily. The dependence on detuning has not been considered in [19-22]. It can be understood in the following way. Absorption by the atomic medium given by Eq. (4) with a drive intensity distribution given by Eq. (5) can be written as

$$
\kappa=\eta\left[\frac{\gamma_{c b}}{|\Omega|^{2}}+\frac{\gamma \omega^{2}}{|\Omega|^{4}}+\left(\frac{\gamma_{c b}}{|\Omega|^{2}}+2 \frac{\gamma \omega^{2}}{|\Omega|^{4}}\right)\left(\frac{x}{L}\right)^{2}\right] .
$$

Then, ratio of the width of the probe intensity distribution to the width of the drive intensity distribution is given by

$$
R=\frac{L}{\Delta x}=\sqrt{\eta z\left(\frac{\gamma_{c b}}{|\Omega|^{2}}+2 \frac{\gamma \omega^{2}}{|\Omega|^{4}}\right)} .
$$

From this we see that the finesse increases with the detuning.

In conclusion, we have performed a proof-of-principle experiment showing that our concept works in $\mathrm{Rb}$ vapor and have experimentally demonstrated the possibility of creating structures having widths smaller than those determined by the diffraction limits of the optical systems. The results obtained here can be viewed as an experimental verification of our approach, as well as evidence supporting the theoretical predictions and results obtained by others [19-22]. The challenges associated with pushing our method to the subwavelength regime are formidable. In our experimental situation, transit-time broadening is the dominant dephasing mechanism that limits the smallness of the region in which a dark state can be formed. Solid-state systems may be more appropriate than a gas. Perhaps the most difficult aspect is devising a way to observe subwavelength structures. This technique might be used in microscopy by studying the distribution of molecules with subwavelength resolution or in lithography by manipulating molecules in the excited state. Also, note that it may be possible to apply this approach to coherent Raman scattering (for example, CARS). This may improve the spatial resolution of CARS microscopy.

\section{ACKNOWLEDGMENTS}

We thank P. Hemmer and O. Kocharovskaya for useful and fruitful discussions, and gratefully acknowledge the support of the Office of Naval Research and the Robert A. Welch Foundation (Grants Nos. A1261 and A1547).
[1] C. A. Mack, Fundamental Principles of Optical Lithography: the Science of Microfabrication (Wiley, West Sussex, England, 2007).

[2] M. O. Scully and K. Druhl, Phys. Rev. A 25, 2208 (1982); U. Rathe and M. O. Scully, Lett. Math. Phys. 34, 297 (1995); M. O. Scully, U. Rathe, C. Su, and G. S. Agarwal, Opt. Commun. 136, 297 (1997).

[3] A. N. Boto, P. Kok, D. S. Abrams, S. L. Braunstein, C. P. Williams, and J. P. Dowling, Phys. Rev. Lett. 85, 2733 (2000).

[4] V. Westphal and S. W. Hell, Phys. Rev. Lett. 94, 143903 (2005).
[5] J. R. Gardner, M. L. Marable, G. R. Welch, and J. E. Thomas, Phys. Rev. Lett. 70, 3404 (1993).

[6] K. D. Stokes, C. Schnurr, J. R. Gardner, M. Marable, G. R. Welch, and J. E. Thomas, Phys. Rev. Lett. 67, 1997 (1991).

[7] J. E. Thomas, Opt. Lett. 14, 1186 (1989); Phys. Rev. A 42, 5652 (1990).

[8] J.-T. Chang, J. Evers, M. O. Scully, and M. S. Zubairy, Phys. Rev. A 73, 031803(R) (2006); J.-T. Chang, J. Evers, and M. S. Zubairy, ibid. 74, 043820 (2006).

[9] A. P. Chu, K. K. Berggren, K. S. Johnson, and M. G. Prentiss, Quantum Semiclassic. Opt. 8, 521 (1996). 
[10] K. S. Johnson, J. H. Thywissen, N. H. Dekker, K. K. Berggren, A. P. Chu, R. Younkin, and M. G. Prentiss, Science 280, 1583 (1998).

[11] J. H. Thywissen and M. G. Prentiss, New J. Phys. 7, 47 (2005).

[12] S. Qamar, S. Y. Zhu, and M. S. Zubairy, Phys. Rev. A 61, 063806 (2000); M. Sahrai, H. Tajalli, K. T. Kapale, and M. S. Zubairy, ibid. 72, 013820 (2005).

[13] P. R. Hemmer, A. Muthukrishnan, M. O. Scully, and M. S. Zubairy, Phys. Rev. Lett. 96, 163603 (2006); Q. Sun, P. R. Hemmer, and M. S. Zubairy, Phys. Rev. A 75, 065803 (2007).

[14] S. E. Harris, Phys. Today 50 (7), 36 (1997).

[15] E. Arimondo, in Progress in Optics, edited by E. Wolf,
(Elsevier Science, Amsterdam, 1996), Vol. XXXV, p. 257.

[16] M. O. Scully and M. S. Zubairy, Quantum Optics (Cambridge University Press, Cambridge, England, 1997).

[17] J. P. Marangos, J. Mod. Opt. 45, 471 (1998).

[18] M. Fleischhauer, A. Imamoglu, and J. P. Marangos, Rev. Mod. Phys. 77, 633 (2005).

[19] M. Kiffner, J. Evers, and M. S. Zubairy, Phys. Rev. Lett. 100, 073602 (2008).

[20] G. S. Agarwal and K. T. Kapale, J. Phys. B 39, 3437 (2006).

[21] A. V. Gorshkov, L. Jiang, M. Greiner, P. Zoller, and M. D. Lukin, Phys. Rev. Lett. 100, 093005 (2008).

[22] D. D. Yavuz and N. A. Proite, Phys. Rev. A 76, 041802(R) (2007). 